\title{
MULTIPLE VISCOUS SOLUTIONS FOR SYSTEMS OF CONSERVATION LAWS
}

\author{
A. V. AZEVEDO AND D. MARCHESIN
}

\begin{abstract}
We exhibit an example of mechanism responsible for multiple solutions in the Riemann problem for a mixed elliptic-hyperbolic type system of two quadratic polynomial conservation laws. In this example, multiple solutions result from folds in the set of Riemann solutions. The multiple solutions occur despite the fact that they all satisfy the viscous profile entropy criterion. The failure of this criterion to provide uniqueness is evidence in support of a need for conceptual change in the theory of shock waves for a system of conservation laws.
\end{abstract}

\section{INTRODUCTION}

Conservation laws have nonunique discontinuous solutions; traditionally this nonuniqueness has been remedied with a series of entropy criteria, designed to pick out the unique physically meaningful solutions. Over the last few years all have failed. On the basis of robust numerical evidence supplemented by mathematical analysis of limiting cases, this paper shows that the most fundamental of all such criteria, the viscous profile criterion, also fails to provide unique solutions for important types of initial data, the Riemann problems.

Riemann problems for systems of two conservation laws of the form

$$
U_{t}+F(U)_{x}=0, \quad x \in \mathrm{RI}, \quad t>0,
$$

are Cauchy problems with initial data given by left and right states:

$$
U(x, t=0)=U_{0}(x)= \begin{cases}U_{l} & \text { if } x<0, \\ U_{r} & \text { if } x>0,\end{cases}
$$

where $U(x, t)=(u(x, t), v(x, t))^{T}$ is a solution of $(1.1)$ for $t>0$ and the function $F$ is a prescribed $C^{2}$ map in $\mathrm{RI}^{2}$. Riemann solutions for a system of conservation laws are formed by sequences of elementary waves, namely shocks and rarefactions, separated by constant states. Rarefactions are scale invariant continuous solutions of the conservation laws, while shocks are discontinuities, which are required to guarantee the existence of solutions of (1.1), (1.2). However, such discontinuities are not uniquely determined by the system. To avoid such nonuniqueness and to select physically meaningful shock waves, the system (1.1) is usually supplemented by the so called entropy criteria.

Received by the editors May 1,1993; originally communicated to the Proceedings of the AMS by James Glimm.

1991 Mathematics Subject Classification. Primary 34D30, 35L65, 35L67, 35L80, 58F14. 
A mechanism which generates multiple Riemann solutions is described in this work, in the context of a system of two conservation laws possessing complex characteristic speeds in a bounded region of points $U=(u, v)^{T}$ in state space. Thus the viscous profile criterion is shown to be insufficient to ensure uniqueness of solutions of (1.1), (1.2), despite the fact that it encompasses and generalizes all other well-known mathematical entropy criteria. The existence of multiple solutions for the model considered here was already established in [2, 3], but the mechanism for the multiplicity was not understood there.

This nonuniqueness is disconcerting for two reasons. The first is that the viscous profile entropy criterion which we employ is the most selective in common usage and takes into account effects usually neglected when physical systems are modeled by conservation laws. The second reason is that this nonuniqueness occurs in a relatively simple mixed-type model with quadratic polynomial flux functions, which captures essential features of Stone's permeability model ([10], [4]). This model is a description of immiscible three-phase flow commonly used in petroleum reservoir engineering. Thus our example provides an indication that Stone's model has multiple solutions. It is not clear which of these solutions is correct, if any.

In order to exhibit the multiplicity, we describe parts of the Riemann solutions, which we found numerically. The solutions occurring in our system of conservation laws may be separated into different classes. The solutions in each class possess analogous sequences of elementary waves. The concept of classes facilitates understanding the mechanism responsible for multiple solutions and helps in establishing the overall Riemann solution for all initial data. In this paper we describe only four classes, although other classes occur in our model. More details can be found in [3]. We believe that separating solutions in classes may be useful in studying other models.

Riemann solutions are usually studied through their projections on planes of initial data $U_{l}$ and $U_{r}$. The solutions we found change class continuously, when the initial data change in $U_{l}$-and $U_{r}$-planes. We detected multiplicities in the Riemann solutions through the formation of a "Whitney tuck" in the set of solutions for a fixed left state. Locally, this set of solutions is a two-dimensional manifold, and the "Whitney tuck" is formed by two-fold curves joining at two singular points, which project onto a "lip" on the $U_{r}$-plane. Thus there are three Riemann solutions for $U_{r}$ inside the lip and one outside.

The plan of this paper is the following. Basic tools are briefly reviewed in $\S 2$. The physical model is presented in $\S 3$. A new kind of shock wave as well as wave curves appearing in this model are presented in $\S 4$ and $\S 5$. The solutions and the mechanisms responsible for multiple solutions are described in $\S 6$. We discuss possible consequences and conceptual remedies in $\S 7$ in the spirit of Glimm and Sharp's proposal [7]. Analytical evidence in support of the existence of the "Whitney tuck" is presented in $\S 8$.

\section{BASIC FACTS}

Riemann problems (1.1), (1.2) are invariant under the scaling $(x, t) \rightarrow$ $(\alpha x, \alpha t)$, for all $\alpha>0$. Therefore we seek scale-invariant solutions, depending on the single variable $\xi=x / t$, which can be represented in state space by curves parametrized by $\xi$. These solutions are constructed by sequences of dis- 
continuous solutions (shocks), continuous solutions (rarefactions) and constant states.

A shock is a discontinuous solution of (1.1) which propagates with speed $s$ and separates two constant states $U_{-}$and $U_{+}$:

$$
U(x, t)= \begin{cases}U_{-} & \text {if } x<s t \\ U_{+} & \text {if } x>s t\end{cases}
$$

it is denoted by $\left(U_{-}, s, U_{+}\right)$, or by $\left(U_{-}, U_{+}\right)$when the speed $s$ is not relevant. The speed $s$ and the states $U_{-}$and $U_{+}$satisfy the Rankine-Hugoniot relation

$$
\mathscr{H}\left(U_{-}, s, U_{+}\right) \equiv-s\left(U_{+}-U_{-}\right)+F\left(U_{+}\right)-F\left(U_{-}\right)=0,
$$

which can be derived from the weak formulation of (1.1); see [17].

Shocks are required in order to ensure existence of solutions of Cauchy problems, but they are not uniquely determined by the partial differential equations. Supplementary conditions, known as entropy criteria, are necessary to select unique physically meaningful solutions. The viscous profile entropy criterion, introduced by Gel'fand [11] and Courant-Friedrichs [9] for strictly hyperbolic systems, takes into account physical effects neglected in the conservation law formulation. It considers (1.1) as an approximation of a parabolic equation of the form

$$
U_{t}+F(U)_{x}=\epsilon\left[D(U) U_{x}\right]_{x}, \quad \epsilon>0,
$$

in the limit as $\epsilon \rightarrow 0^{+}$, where $D(U)$ is a $2 \times 2$ viscosity matrix determined by small scale physical effects. This matrix is assumed to be positive definite [8]. A shock wave is considered admissible, or to have a viscous profile, if it is the limit of a solution of (2.2) of the form

$$
U=U(\zeta), \quad \zeta=(x-s t) / \epsilon,
$$

satisfying

$$
\lim _{\zeta \rightarrow-\infty} U(\zeta)=U_{-} \text {and } \lim _{\zeta \rightarrow \infty} U(\zeta)=U_{+} .
$$

This solution is called a traveling wave with speed $s$. Using (2.3) and (2.4) in (2.2), it is easy to show that the shock $\left(U_{-}, s, U_{+}\right)$is admissible if and only if there exists an orbit from $U_{-}$to $U_{+}$of the associated dynamical system

$$
\dot{U}=[D(U)]^{-1}\left[-S\left(U-U_{-}\right)+F(U)-F\left(U_{-}\right)\right],
$$

where the dot denotes differentiation with respect to $\zeta$. Thus $U_{-}$and $U_{+}$are singularities of (2.5) and they lie on the Hugoniot curve. For each $U_{-}$, this curve is obtained by eliminating $s$ in $(2.1)$; it bifurcates topologically when $U_{-}$ crosses certain loci, namely the secondary bifurcation locus and the coincidence locus [14].

Taking into account different types of singularities of the dynamical system (2.5), admissible shock waves of interest can be classified as follows: 1-shocks, where $U_{-}$is a repeller and $U_{+}$a saddle; 2-shocks, where $U_{-}$is a saddle and $U_{+}$an attractor; transitional shocks, where $U_{-}$and $U_{+}$are saddles. The set of right states $U$ which can be connected to a left state $U_{-}$by an $i$-shock is denoted by $S_{i}\left[U_{-}\right]$. The limit cases of $i$-shocks, when the nodes become saddle-nodes, are also considered $i$-shocks $(i=1,2)$ in the present work. 
These limits are called sonic shocks. There are other types of discontinuities which will not be used in this paper. We recall that Lax's entropy criterion for shocks defines 1-shocks and 2-shocks by the type of singularities but without taking into account the existence of a connecting orbit between them [17]. See also [9]. Thus the viscous profile entropy criterion encompasses Lax's [17] and Oleinik's [20] entropy criteria.

The other elementary waves occurring in Riemann solutions are the rarefactions. In physical space (the set $\{(x, t): x \in \mathrm{RI}, t>0\}$ ), rarefactions are represented by fans of characteristic curves. In state space, rarefactions are constructed using the part of the integral curves of the eigenvector line field $r_{i}(U)$ associated to the eigenvalue $\lambda_{i}(U)$ of the matrix $d F(U)$, taking into account that $\lambda_{i}(U)=\xi=x / t$ (where $i=1,2$ and $\left.\lambda_{1}(U)<\lambda_{2}(U)\right)$. An $i$-rarefaction curve $R_{i}\left[U_{-}\right]$from $U_{-}$, associated with the family $i$, is the part of the integral curve of $r_{i}(U)$ starting at $U_{-}$, along which $\lambda_{i}$ increases. For quadratic polynomial flux functions rarefactions are studied in detail in [18].

\section{THE MODEL}

The type of a conservation law system is determined by the behavior of the eigenvalues of $d F(U)$. A system is of (elliptic-hyperbolic) type if there exists a region $E$ in state space (the elliptic region) for which the eigenvalues are complex conjugate inside and real distinct outside.

Systems of conservation laws of mixed elliptic-hyperbolic type arise in the theory of three-phase flow in porous media (for example in Stone's permeability model) [4], widely employed in petroleum reservoir engineering [10]. They arise in many other applications as well, including transonic flow in gas dynamics [9].

With $\rho$ being a positive constant, we consider the flux function given by

$$
F(u, v)=\left[\begin{array}{c}
\frac{1}{2}\left(-u^{2}+v^{2}\right)+\rho v \\
u v-\rho u
\end{array}\right]
$$

For the model (3.1) $E$ is a circle of radius $\rho$ with the center at the origin. The model was proposed and studied by Holden in [13] without taking into account the viscous profile criterion; the model is a perturbation of Symmetric Case I defined in [21]. Furthermore, for simplicity, we take the viscosity matrix $D(U)$ in (2.2) to be the identity. In our work, we denote the right-hand side of (2.5) with $D=I$ by $\mathscr{X}_{s}=\mathscr{X}_{s}\left(U_{-}, U\right)$, which is a vector field depending on three parameters $U_{-}$and $s$.

In this model, the coincidence locus (the locus of point $U$ such that $\lambda_{1}(U)=$ $\left.\lambda_{2}(U)\right)$ is the boundary of $E$. For $U_{-}$outside $E$, the Hugoniot curve locally has two branches at its primary bifurcation point $U_{-}$. For $U_{-}$on the coincidence locus, the two branches collapse; for $U_{-}$inside $E$, the Hugoniot curves become the union of an isolated point $U_{-}$and detached branches outside $E$. Secondary bifurcations for the Hugoniot curve occur at three straight lines $B_{1}=\{(u, v) \mid v=\rho\}, B_{2}=\{(u, v) \mid v=\sqrt{3} u-2 \rho\}$ and $B_{3}=\{(u, v) \mid v=$ $-\sqrt{3} u-2 \rho\}$, which form an equilateral triangle with edges tangent to the circle $E$. These sets are shown in Figure 4.3.

The construction of solutions of the Riemann problem for (3.1) is simplified by the three-fold symmetry property [13]. Namely, the following proposition holds. 
Proposition 3.1. If $U(x, t)$ solves (1.1) and (1.2), with $F$ given by (3.1), then $O_{ \pm} U$ also solves (1.1) with initial value $O_{ \pm} U_{0}(x)$, where $O_{ \pm}$denotes a rotation by $\pm \frac{2 \pi}{3}$.

The verification of the existence of orbits connecting the singularities was performed numerically. An essential fact to ensure that numerical verifications were trustworthy is that $\mathscr{X}_{s}$ does not have closed orbits for $s \neq 0$. This fact can be proved using Bendixson's criterion [1]. For $s=0, \mathscr{X}_{s}$ reduces to Hamiltonian systems studied in detail by Frommer and Bautin [23]; we utilize several of their results to draw some of our conclusions.

\section{TRANSITIONAL SHOCKS}

Transitional shock waves are not associated to any specific family. They are represented by orbits connecting two saddles of the dynamical system (2.5). For quadratic gradient vector fields, Chicone's theorem [6] ensures that such orbits lie on straight lines. In our model, $\mathscr{X}_{s}$ is not a gradient vector field, unless $\rho=0$, and we identify transitional shocks possessing orbits not lying on a straight line (see Figure 4.1). The existence of these shocks can be proved utilizing perturbations of the Hamiltonian systems studied in [23]. Therefore, in our model transitional shocks can be separated in two types: shocks $T_{s}$ with connecting orbits lying on straight lines (straight transitional shocks) and shocks $T_{c}$ with connecting orbits lying outside any straight line (curved transitional shocks).

In our model, straight transitional shocks can be shown to have orbits lying on the secondary bifurcations $B_{1}, B_{2}$ and $B_{3}$, see [14].

Curved transitional shock waves $T_{c}$ are important new shock waves responsible for a change of admissibility of other shock waves. For example, if $\left(U_{-}, s\right)$ corresponds to $\mathscr{Z}_{s}$ with configuration shown in Figure 4.2, the shock between the saddle $U_{-}$and the node $U_{+}^{\prime}$ is an admissible 2-shock. Fixing $U_{-}$and changing $s$ until the configuration of $\mathscr{X}_{s}$ becomes similar to that in Figure 4.1, the connection between the saddle and the node is broken and the shock $\left(U_{-}, s, U_{+}^{\prime}\right)$ ceases to be admissible.

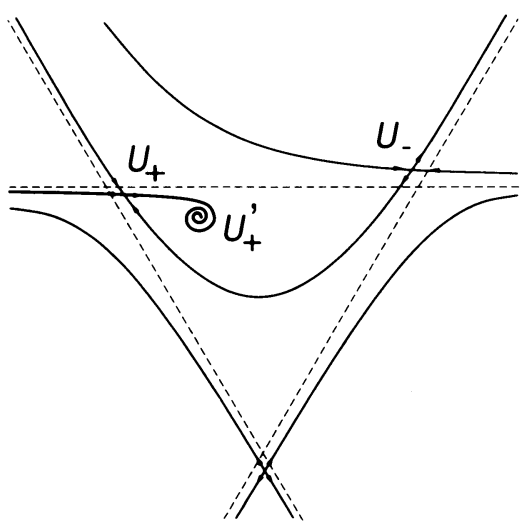

Figure 4.1. Saddles connected by a curved orbit.

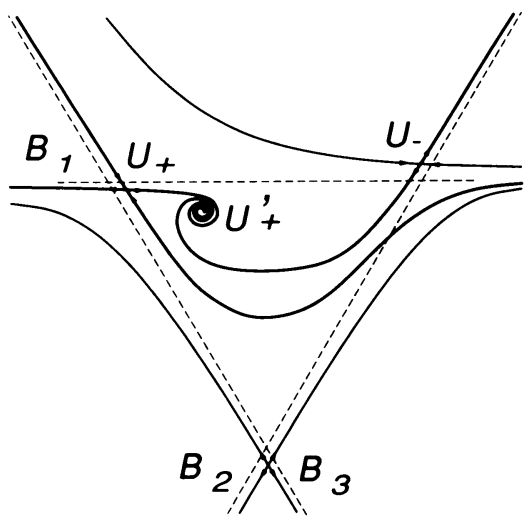

FIGURE 4.2. A configuration of $X_{s}\left(U_{-} . \cdot\right)$ associated to a 2 -shock. 


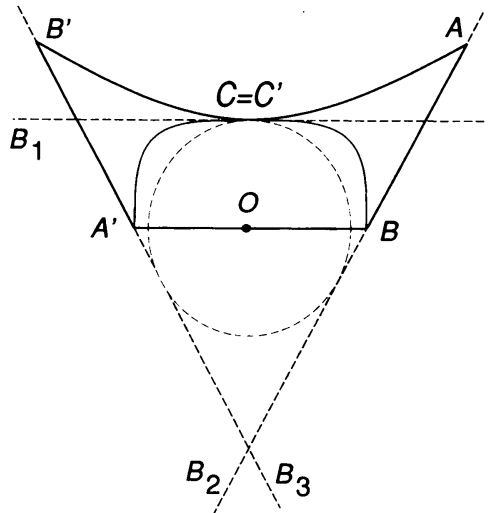

FIGURE 4.3. Sets of saddles $U_{-}, U_{+}$which are connected by a curved orbit.

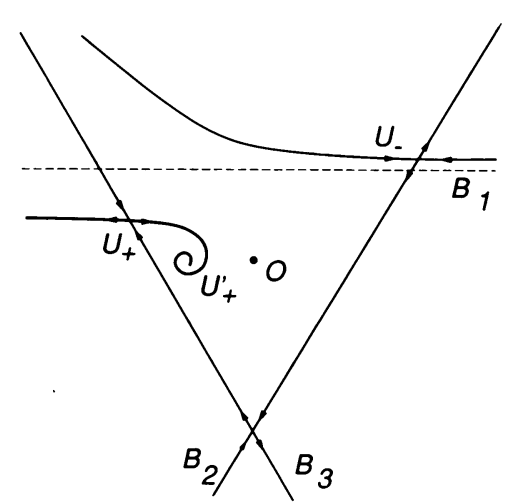

FIgURE 4.4. Configuration of $\mathscr{Z}_{s}\left(U_{-}, \cdot\right)$ for $U_{-}$on $A B$.

We identified a set of points $\left(U_{-}, s\right)$ in parameter space for which $\mathscr{X}_{s}\left(U_{-},.\right)$ has a curved orbit connecting the saddle $U_{-}$to another saddle. The region $A B C$ shown in Figure 4.3 consists of projection of the above mentioned set on the $U_{-}$-plane. The region $A^{\prime} B^{\prime} C^{\prime}$ in Figure 4.3 is the projection on the $U_{+}$-plane of the set of points $\left(U_{+}, s\right)$ for which $\mathscr{Z}_{s}\left(., U_{+}\right)$has a curved orbit connecting another saddle to the saddle $U_{+}$. In Figure 4.3, the secondary bifurcation locus is the three dashed lines and the coincidence locus is the dashed circle. The boundary $A C$ is defined as the $U_{-}$- projection of the set of points $\left(U_{-}, s\right)$ with $U_{+}$being a saddle-node lying on $A^{\prime} C^{\prime}$ for which one of the strong invariant manifolds has the saddle $U_{-}$as its limit point. The boundary $B C$ is defined in an analogous way, except that $U_{-}$is the saddle-node while $U_{+}$is the saddle (see [3]). We call the curves $A C$ and $B C$ minus transitional boundaries and the curves $C^{\prime} A^{\prime}$ and $C^{\prime} B^{\prime}$ plus transitional boundaries.

In Figure 4.3, the line $A B$ is defined as the $U_{-}$-projection of points $\left(U_{-}, s\right)$ with associated dynamical system $\mathscr{X}_{s}$ having a configuration as shown in Figure 4.4. The region $A^{\prime} B C$ is the $U_{+}^{\prime}$-projection of the set of points $\left(U_{+}^{\prime}, s\right)$ for which $\mathscr{X}_{s}\left(., U_{+}^{\prime}\right)$ has saddles connected by a curved orbit and $U_{+}^{\prime}$ is a node. The line $A^{\prime} B$ is defined as the $U_{+}$-projection of points $\left(U_{+}^{\prime}, s\right)$ with $\mathscr{Z}_{s}$ having configuration as in Figure 4.4, $U_{-}$lying on the segment $A B$ and $U_{+}^{\prime}$ being the node.

The point $A$ in Figure 4.3 plays a special role in the Riemann solutions for the model (3.1). In the context of dynamical systems, it is the value of $U_{-}$on $B_{2}$ for which there exists an $s$ with $\mathscr{Z}_{s}(A$, .) having a configuration which is the limit of that shown in Figure 4.4. In this case, $U_{+}^{\prime}$ tends to $U_{+}$, becoming $A^{\prime}$ in the limit (Figure 4.3), i.e., $A^{\prime}$ is a saddle-node and the singularities $A$ and $A^{\prime}$ are part of a graph consisting of two straight line orbits which join at a singularity located at the intersection of $B_{2}$ and $B_{3}$. Also, $A$ is the intersection of the secondary bifurcation $B_{2}$ with the transitional boundary $A C$. The coordinates of $A$ and $A^{\prime}$ are $\left(\frac{14+2 \sqrt{3}}{\sqrt{3}} \rho,(12+2 \sqrt{3}) \rho\right)$ and $\left(-\frac{2}{\sqrt{3}} \rho, 0\right)$ respectively. The curves $A C$ and $B C$ are the loci where a transitional shock 
$T_{c}$ changes into a limit of 1 - or 2-shocks (the sonic shock waves), while $A B$ is the locus where $T_{c}$ changes into a sequence of two straight transitional shocks, one lying on $B_{2}$ and the other on $B_{3}$.

To summarize, there are two injective maps defined on the domain $A B C$. The first is a map from $A B C$ onto $A^{\prime} B^{\prime} C^{\prime}$ : given a saddle $U_{-}$within $A B C$, it associates another saddle $U_{+}$in $A^{\prime} B^{\prime} C^{\prime}$ connected to $U_{-}$by a curved orbit, as shown in Figure 4.1. The second is a map from $A B C$ to $A^{\prime} B C$ : given any saddle $U_{-}$within $A B C$, it associates the node $U_{+}^{\prime}$ shown in Figure 4.1. The first map is called the curved transitional map $\left(\mathscr{T}_{c}\right)$ and the second one, the curved node map $\left(\mathscr{N}_{c}\right)$.

Although Figures 4.1 to 4.4 show only configurations with attractor-nodes, by symmetry it is possible to obtain other configurations with repeller-nodes.

Dynamical systems for quadratic polynomial flux functions were studied in detail in [5]. Transitional shock waves with curved orbits were first observed in this model in [3]; they have an important role to ensure the continuous dependence of a Riemann solution with respect to initial data. The stability of transitional shock waves associated both to curved and to straight orbits was studied numerically in [24].

\section{WAVE CURVES}

The principal task in the construction of Riemann solutions is to build wave curves in state space. Wave curves parametrize wave groups. A wave group is a solution of (1.1) and (1.2) composed of a finite number of shocks and rarefactions with no embedded sectors of constant states, which satisfies

$$
U(\xi)=U_{-}, \text {for } \xi<\xi_{-} \text {and } U(\xi)=U_{+} \text {, for } \xi>\xi_{+} \text {, }
$$

where $U_{-}$and $U_{+}$are constant states, and $\xi_{-}$and $\xi_{+}$are arbitrary real numbers. The states $U_{-}$and $U_{+}$are called initial and final states of the wave group, respectively. If a wave group contains only $i$-rarefactions and $i$-shocks it is called an $i$-wave group. A solution of a Riemann problem consists of $i$-wave groups, transitional shocks and intermediate states, with wave speeds increasing from left to right. The $i$-wave curve based on (or from) $U_{-}$(denoted by $\left.\mathscr{W}_{i}\left[U_{-}\right]\right)$is the set of all $i$-wave groups with initial state $U_{-}$.

In our model, Riemann solutions are obtained by sequences of a 1-wave group starting at $U_{l}$, a number of transitional shocks and a 2-wave group ending at $U_{r}$. The 2-wave curves have only a single connected branch containing its base point, while the 1-wave curves have a connected branch containing its base point and one or two detached branches. The branches containing their base points are called local and the other non-local. Figure 5.1 and Figure 5.2 show examples of local branches of 1-wave curves and 2-wave curves respectively, while Figure 5.3 shows examples of non-local branches of 1-wave curves. We observe that $\mathscr{W}_{2}\left[U_{3}\right]$ stops at a point inside $E$, because the segment of 2shock curves from $U_{3}$ is not admissible beyond that point. We do not show the internal structure of each wave curve; it can be found in [3] and [13]. In Figures 5.1-5.3 the points $U_{1}, U_{2}, U_{3}$ and $U_{4}$ are base states for the wave curves displayed. Other wave curves can be obtained by rotation, since this model satisfies Proposition 3.1. 


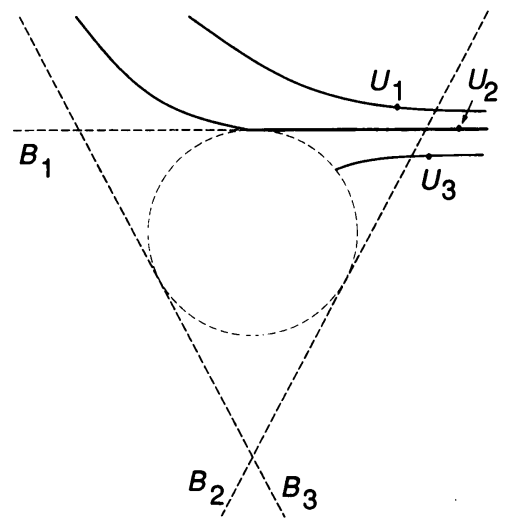

Figure 5.1. Examples of 1-wave curves.

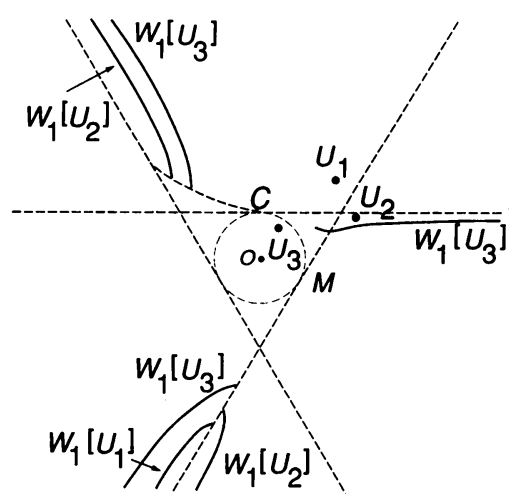

Figure 5.3. Examples of nonlocal branches of 1-wave curves.

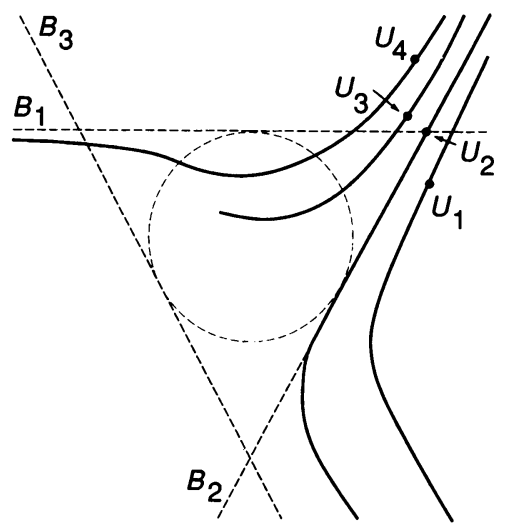

Figure 5.2. Examples of 2-wave curves.

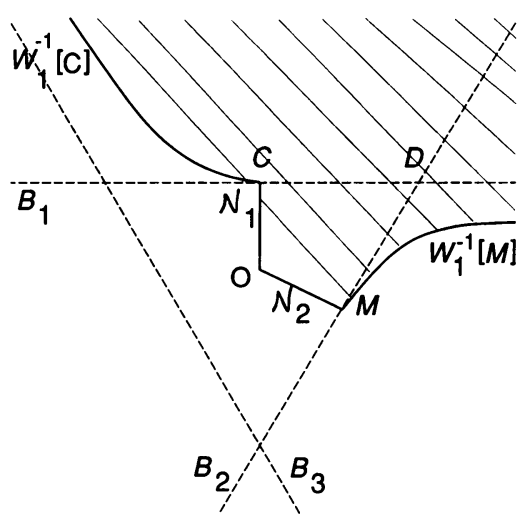

FIgURE 6.1. The third part of the $U_{l}$-plane.

\section{Multiple Solutions}

Riemann solutions for systems of two conservation laws are usually described utilizing projections on the $U_{l}$ and $U_{r}$ planes. In general, this is done by fixing a point $U_{l}$ in a certain region of the left state plane and studying the behavior of the solutions for all states $U_{r}$ in the right state plane. However, we will see that this strategy has difficulties inherent to the usage of projections.

In this section we present the solutions of Riemann problem (1.1), (1.2), (3.1), for $U_{l}$ lying in certain neighborhoods. These solutions satisfy the requirement that speed increases from left to right, verified by combining numerical studies with the usage of the triple shock rule [14].

Because of the three-fold symmetry (see Proposition 3.1), it suffices to describe Riemann solutions for $U_{l}$ in one third of the state space, which may be chosen as the region including part of $B_{2}$ shown in Figure 6.1. In this figure, 
$\mathscr{W}_{1}^{-1}[P](P=C, M)$ are the curves formed by the states $U_{l}$ which can be connected to $P$ by $\mathscr{W}_{1}\left[U_{l}\right]$, while $\mathscr{N}_{1}$ and $\mathscr{N}_{2}$ are the images through $\mathscr{N}_{c}$ of segments $D C$ and $D M$ respectively. Experiments indicate that $\mathscr{W}_{1}\left[U_{l}\right]$ in this region intersect the secondary bifurcation locus $B_{2}$. We denote these points by $U_{n}$. The state $U_{n}$ is a constant intermediate state in the Riemann solution.

Riemann solutions for model (3.1) may be grouped into eight distinct classes, which differ through the number or type of transitional shocks present. Class I consists of solutions formed by a 1-wave group, a transitional shock $T_{s}$ and a 2-wave group. Class II consists of a 1-wave group followed by a 2 -wave group. Class III solutions are formed by a 1-wave group, a curved transitional shock $T_{c}$ and a 2-wave group. Class IV consists of a sequence of two transitional shocks $T_{s}$, between a 1-wave group and a 2-wave group. In this paper we describe only these four classes, although other classes occur in this model. In all the solutions described, wave groups and transitional shocks are separated by constant states. All classes may contain degenerate cases where the 1-wave group or the 2-wave group vanish. We remark that only Classes I and II have already appeared in earlier works (for example, see [21]).

Using the continuous dependence on $U_{l}$ and $U_{r}$, it is easy to verify that the solution in each class deforms $L_{l o c}^{1}$-continuously into a solution belonging to a neighboring class. Such "adjacent" classes are indicated by Diagram 6.1.

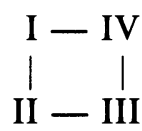

Diagram 6.1. Connection of some classes.

For this model, when $\rho=0$, or $\rho>0$ and $U_{n}$ is sufficiently far away from the origin, the Riemann solution lies in Classes I and II, depending on where $U_{r}$ is. For $\rho>0$ with $U_{n}$ lying above the point $A$ defined in the previous section, Figure 6.2 shows subdivisions of the $U_{r}$-plane in regions according to the class of the corresponding solution. In that figure, point 1 is the boundary between the sets of points on $B_{2}$ representing 2-shocks and straight transitional shocks $T_{s}$; it satisfies the inequalities $\lambda_{1}\left(U_{n}\right)<s\left(U_{n}, 1\right)<\lambda_{2}\left(U_{n}\right)$ and $\lambda_{1}(\mathbf{1})<s\left(U_{n}, \mathbf{1}\right)=\lambda_{2}(\mathbf{1})$. Also, point 2 is the boundary between points on $B_{2}$ representing straight transitional shocks $T_{s}$ and non-local 1-shocks; it satisfies the conditions $\lambda_{1}\left(U_{n}\right)=s\left(U_{n}, 2\right)<\lambda_{2}\left(U_{n}\right)$ and $\lambda_{1}(2)<s\left(U_{n}, 2\right)<\lambda_{2}(2)$. For this range of $U_{l}$ and all states $U_{r}$, there is exactly one Riemann solution, which was obtained in [21]. We remark that the curve $\Lambda$, shown in Figure 6.2, is the limit of a segment of $\mathscr{W}_{2}\left[U_{l}^{\prime}\right]$ for $U_{l}^{\prime}$ tending to $U_{n}$ from the left-hand side of $B_{2}$, and that the rarefaction $R_{2}[1]$ is also the limit of a segment of $\mathscr{W}_{2}\left[U_{l}^{\prime \prime}\right]$ for $U_{l}^{\prime \prime}$ tending to $U_{n}$ from the right-hand side of $B_{2}$. If $U_{r}$ lies in the region II above region $\mathrm{I}$, the solution is a 1-wave group from $U_{l}$ to an intermediate state on the local branch of $\mathscr{W}_{1}\left[U_{l}\right]$, followed by a 2-wave group to $U_{r}$. If $U_{r}$ lies in region II below $\mathrm{I}$, the solution is a 1-wave group from $U_{l}$ to an intermediate state on the non-local branch of $\mathscr{W}_{1}\left[U_{l}\right]$ followed by a 2-wave group. If $U_{r}$ lies in region $\mathrm{I}$, the solution is a 1-wave group from $U_{l}$ to $U_{n}$, a transitional shock $T_{s}$ from $U_{n}$ to a state on $B_{2}$ between 1 and 2, followed by a 2-wave group to $U_{r}$. 


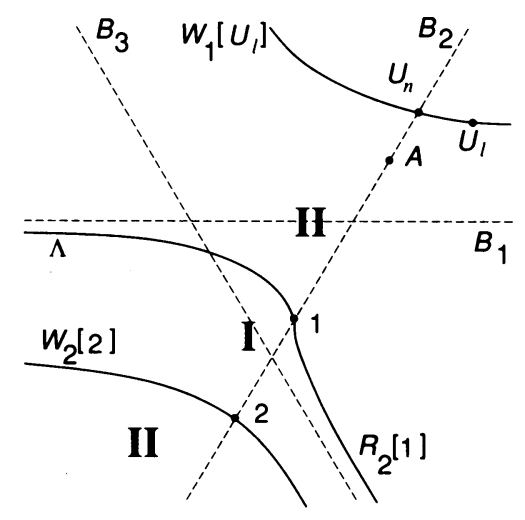

FIGURE 6.2. $U_{r}$-plane for $U_{n}$ above $A$.

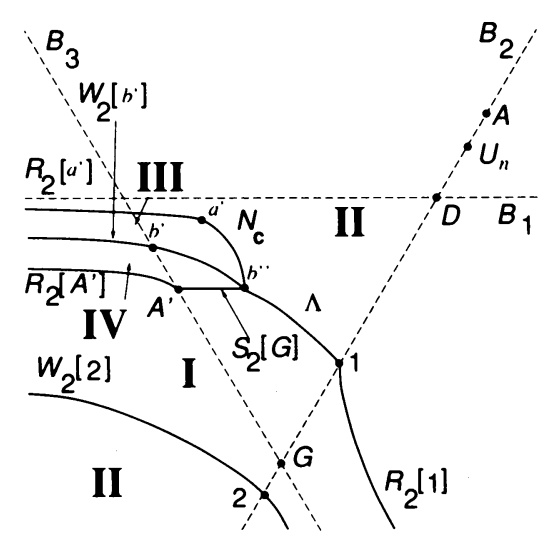

FIGURE 6.4. $U_{r}$-plane for $U_{n}$ below but close to $A$.

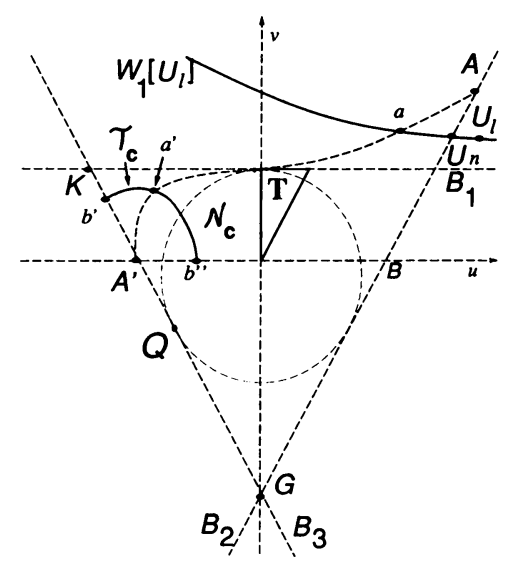

Figure 6.3. The curves $\mathscr{T}_{c}\left[\mathscr{W}_{1}\left[U_{l}\right]\right]$ and $\mathscr{N}_{c}\left[\mathscr{W}_{1}\left[U_{l}\right]\right]$.

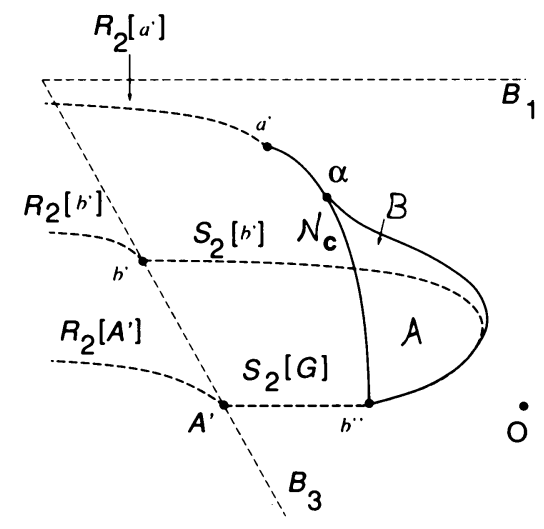

Figure 6.5. The regions $\mathscr{A}$ and $\mathscr{B}$, for $U_{n}$ lying between $A$ and $D$.

If $U_{n}$ lies on $B_{2}$, underneath but close to $A$, the intersection of the local branch of $\mathscr{W}_{1}\left[U_{l}\right]$ with the region $A B C$ is the segment between points $a$ and $U_{n}$ (see Figure 6.3). The images of the segment $\left[a, U_{n}\right]$ under the two curved transitional maps are denoted by $\mathscr{T}_{c}$ and $\mathscr{N}_{c}$ in Figures 6.3, 6.4 and 6.7. These two curves join at a point $a^{\prime}$, where they are tangent. Curve $\mathscr{T}_{c}$ ends at a point $b^{\prime}$ on $B_{3}$, which is the intersection of $B_{3}$ with the Hugoniot curve from $U_{n}$. Denoting by $G$ the intersection of $B_{2}$ and $B_{3}, b^{\prime}$ is also the image of $U_{n}$ by a sequence of two transitional shocks $T_{s}\left(U_{n}, s_{1}, G\right)$ and $\left(G, s_{2}, b^{\prime}\right)$, $s_{1}<0<s_{2}$ (see the configuration in Figure 4.4). The curve $\mathscr{N}_{c}$ ends at the point $b^{\prime \prime}$ on $A^{\prime} B$, which is a node as shown in Figure 4.4.

Consider a curve based on $U_{l}$ which intersects $B_{2}$ at a fixed point $U_{n}$. If $U_{l}$ lies on the right-hand side of $B_{2}$, the segment of $\mathscr{W}_{1}\left[U_{l}\right]$ that lies within $A B C$ is just a rarefaction curve through $U_{n}$; thus the shape of $\mathscr{W}_{l}\left[U_{l}\right]$ in this region is independent of $U_{l}$ provided $U_{n}$ is kept fixed. On the other hand, if 
$U_{l}$ lies on the left-hand side of $B_{2}$, within $A B C$ the wave curve is a shock curve through $U_{n}$, whose shape necessarily changes if $U_{l}$ is changed, even if $U_{n}$ is kept fixed. Thus, near $a^{\prime}$ the dependence on $U_{l}$ of the shape of the images $\mathscr{T}_{c}$ and $\mathscr{N}_{c}$ of $\mathscr{W}_{1}\left[U_{l}\right]$ has a different behavior for $U_{l}$ on each side of $B_{2}$.

Figure 6.4 shows the solution classes for $U_{n}$ below but close to $A$, and for $U_{r}$ in the regions displayed. Points 1 and 2 are defined as in Figure 6.2. The point $A^{\prime}$ is the boundary on $B_{3}$ between the segments of 2-shocks and of transitional shocks from $G$. If $U_{r}$ lies in regions I and II, the solutions are analogous to those described for $U_{n}$ above $A$ in Figure 6.2. If $U_{r}$ lies in region III, the solution is a sequence of the following waves: a local 1-wave group from $U_{l}$ to a state $U_{m}$ lying on the segment of wave curve $\left[a, U_{n}\right]$ of Figure 6.3, a $T_{c}$ shock from $U_{m}$ to $U_{m_{1}}$ on $\mathscr{T}_{c}$ and a 2-wave group from $U_{m_{1}}$ to $U_{r}$. If $U_{r}$ lies in region IV, the solution consists of a 1-wave group from $U_{l}$ to $U_{n}$ on $B_{2}$, a $T_{s}$ shock from $U_{n}$ to $G$, another $T_{s}$ from $G$ to an intermediate state on $B_{3}$ between $A^{\prime}$ and $b^{\prime}$, and finally a 2-wave group to $U_{r}$.

When $U_{l}$ moves down, the segment bounded by $b$ and $b^{\prime \prime}$ (which consists of shocks) moves towards $\mathscr{N}_{c}$ and $\mathscr{W}_{2}\left[b^{\prime}\right]$ intersects $\mathscr{N}_{c}$ at another point besides $b^{\prime \prime}$. This gives rise to two regions $\mathscr{A}$ and $\mathscr{B}$ in the $U_{r}$-plane (Figure 6.5). For $U_{r}$ in $\mathscr{A}$ or $\mathscr{B}$ there are three distinct solutions. For any state $U_{r}$ in $\mathscr{A}$, there is one solution in Class II, one in Class III and one in Class IV. For $U_{r}$ in $\mathscr{B}$, there is one solution in Class II and two other solutions in Class III. The two Class III solutions for $U_{r}$ in region $\mathscr{B}$ exist because 2-shock curves with two distinct base points on $\mathscr{T}_{c}$ intersect at the same $U_{r}$. For any point $U_{r}$ in region $\mathscr{A}$, Class III and Class IV solutions exist because there are a 2-shock curve based on $\mathscr{T}_{c}$ and a 2 -shock curve based on $B_{3}$ which intersect at $U_{r}$. The regions $\mathscr{A}$ and $\mathscr{B}$ are the projections of a topological structure similar to a Whitney tuck [21] in Figure 6.6, where the horizontal plane is the $U_{r}$-plane and the vertical axis $z$ is an arc length parametrization along $B_{3}$ and $\mathscr{T}_{c}$. It increases from $A^{\prime}$ to $b^{\prime}$ on $B_{3}$, and then from $b^{\prime}$ to $a^{\prime}$ on $\mathscr{T}_{c}$. The surface of $\left(U_{r}, z\right)$-points in Figure 6.6 provides a geometric representation of the solutions for a fixed $U_{l}$ in this range; it is obtained by lifting to the space $(u, v, z)$ the dashed Hugoniot curves constructed in Figure 6.7.

The set of Riemann solutions for fixed $U_{l}$ is locally a two-dimensional manifold, and the "Whitney tuck" is formed by a pair of fold curves joining at two singular points. The "Whitney tuck" projects onto the union of $\mathscr{A}$ and $\mathscr{B}$. The boundaries of this union are the $U_{+}$-projection of the folds. We have preliminary indications that our "Whitney tuck" is continuous, and smooth except at the fold curve corresponding to $\left[\alpha, \mathscr{N}_{c}, b^{\prime \prime}\right]$ and at the Hugoniot curve $\left[b^{\prime}, b^{\prime \prime}\right]$ in Figure 6.7. The singularity $\alpha$ seems to be a cusp where the folds meet tangentially; however, the two fold curves meet transversally at $b^{\prime \prime}$.

If $U_{l}$ continues to move downwards so that $U_{n}$ tends to point $D$ (the intersection of $B_{1}$ and $\left.B_{2}\right), \mathscr{A}$ increases while $\mathscr{B}$ decreases. Eventually $\mathscr{B}$ disappears for $U_{n}$ on $D$ and $\mathscr{A}$ becomes a triangle $\mathrm{T}$ bounded by $B_{1}$, the $v$ axis and a Hugoniot curve segment along a line parallel to $B_{2}$ across the origin.

Numerical evidence indicates that $\mathscr{A}$ and $\mathscr{B}$ start appearing simultaneously at $b^{\prime \prime}$. More precisely, there is a curve in the $U_{l}$-plane separating the $U_{l}$-set for which $\mathscr{A}$ and $\mathscr{B}$ exist from the $U_{l^{-}}$-set for which they do not exist. This $U_{l^{-}}$boundary is the set of $U_{l}$ for which $\mathscr{N}_{c}$ and a certain envelope $\mathscr{E}$ are tangent 


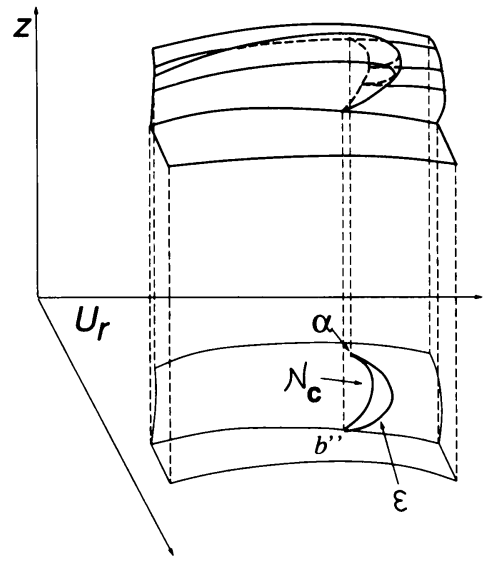

FIGURE 6.6. The Whitney tuck.

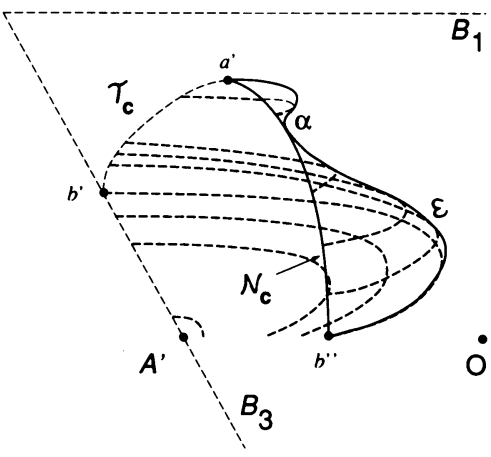

FIGURE 6.7. The tangency of the envelope with $\mathscr{N}_{c}$.

at $b^{\prime \prime}$. This envelope is formed by segments of 2-shock curves with left states on $\mathscr{T}_{c}$. For $U_{l}$ below this boundary $\mathscr{A}$ and $\mathscr{B}$ exist: the envelope and $\mathscr{N}_{c}$ are tangent at a certain point $\alpha$ on $\mathscr{N}_{c}$ between $a^{\prime}$ and $b^{\prime \prime}$ (see Figure 6.7). This point $\alpha$ is the intersection of $\mathscr{N}_{c}$ and $\mathscr{E}$ in Figure 6.5. For $U_{l}$ on this boundary $\alpha$ coincides with $b^{\prime \prime}$. In Figure 6.7, the segments of 2-shock curves with left states on $\mathscr{T}_{c}$ are drawn as thick dashed curves, the envelope is shown by a solid curve and $\mathscr{N}_{c}$ by another solid curve. The region above $\alpha$ which is bounded by the envelope $\mathscr{E}$ and by $\mathscr{N}_{c}$ is formed by segments of 2 -shock waves which cannot be used in the Riemann solution. This is so because for $U_{r}$ on these segments the speed of the $T_{c}$ shock from the intermediate state $U_{m}$ on $\mathscr{W}_{1}\left[U_{l}\right]$ to the other intermediate state $U_{m_{1}}$ on $\mathscr{T}_{c}$ is greater than the speed of the 2-shock from state $U_{m}$ to $U_{r}$. Thus, the region mentioned above does not appear in Figure 6.5.

\section{Discussion}

The most important conclusion in this work is that the viscous profile criterion is insufficient to guarantee the uniqueness of Riemann solutions. In our particular example, the loss of uniqueness seems to be related to an envelope of a shock curve family present in the Riemann solution, as well as to a change of class in the solution along another curve.

One would hope that the lack of uniqueness could be remedied by requiring that the whole Riemann solution be the limit of solutions of parabolic equations. This would be a stronger requirement than the one we employ, which forces each individual wave to be such a limit. However, we do not expect that such a requirement would resolve the nonuniqueness.

We speculate that to resolve the nonuniqueness, rather than strengthening the entropy criterion, a conceptual change in the meaning of Riemann solutions is needed. In essence, one has to take into account information present in the continuous initial data given by the physics of the problem, but disregarded in the scale invariant discontinuous Riemann data (1.2).

Consider the conservation laws (1.1), modified by the inclusion of parabolic 
terms, with a smooth initial data $U_{0}(x)$ coinciding with the Riemann data (1.2) outside a compact interval. Such a parabolic initial-value problem should have a unique solution; it should tend to a scale-invariant solution of the Riemann problem (1.1), (1.2) for large times. Thus Riemann solutions represent asymptotic solutions rather than solutions of initial-value problems. Under certain conditions, the asymptotic solution of the initial-value problem may be independent of the particular interpolation between $U_{L}$ and $U_{R}$ contained in the initial data. In such situations, one would hope that the Riemann problem would have unique solutions. However, in general the detailed information contained in the interpolation between $U_{L}$ and $U_{R}$ should influence the asymptotic solution. From this perspective, non-unique Riemann solutions should be the rule rather than the exception. We know at least of two earlier examples ([16], [18] ) which illustrate the need for distinguishing asymptotic (Riemann) solutions from solutions of initial-value problems.

The same ideas may be expressed in the language of scattering theory; we outline this approach, following Glimm and Sharp [7]. In scattering theory, it is postulated that one can measure only incoming waves ( from $t \rightarrow-\infty$ ) and outgoing waves ( at $t \rightarrow \infty$ ). The $S$ "matrix" is a mapping from the incoming to the outgoing waves, which contains in a synthetical way the large scale dynamics of the system. For (nonlinear) physical systems, the principle of causality indicates that $S$ should be a well-defined (nonlinear) map.

For time-reversible physical processes, such as in Quantum Mechanics, the so called time-dependent approach to scattering theory relies on the construction of two mappings $W^{+}$and $W^{-}$, the wave operators. $W^{+}$maps the $t=0$ initial data onto the $t \rightarrow \infty$ asymptotic solution, or outgoing waves. $W^{-}$maps the $t=0$ initial data onto the $t \rightarrow-\infty$ asymptotic solution it originated from, or incoming waves. Thus, we essentially have $S=W^{+}\left(W^{-}\right)^{-1}$.

However, conservation laws usually are employed to describe irreversible processes; thus $W^{-}$does not exist, and one cannot define $S$ in terms of $W^{+}$and $W^{-}$. Glimm and Sharp [7] propose that $S$ rather than $W^{+}$is fundamental for scattering. Scale invariance can be imposed on outgoing states and perhaps on incoming ones, but there is no reason in Physics or Mathematics to require this of time zero states, i.e. during the wave interaction period. Thus we obtain a well-defined $W^{+}$operator by requiring its domain to consist of continuous functions. It remains an open question to specify the minimum information on the incoming waves to give uniquely determined outgoing ones, such as scale invariant data for $\left(W^{-}\right)^{-1}$.

\section{APPENDIX}

The analysis of the folding mechanism responsible for multiple solutions in the "lip region" is complicated and has not been carried through for general $U_{l}$. However, there is a limit case which can be analyzed, for $U_{l}$ on the secondary bifurcation locus $B_{1}$. In this limit, we describe explicitly the Riemann solution surface of Figure 6.6.

For a set of pairs $U_{l}, U_{r}$, multiple Riemann solutions occur as described in $\S 6$ because $U_{r}$ can be reached in state space by three distinct 2-shock curves, all intersecting at $U_{r}$. Each of these 2-shock curves is based on a distinct intermediate state, representing a region of constant solution in physical space. In 
state space, these three intermediate states are points on two connected curves: $\mathscr{W}_{1}\left[U_{l}\right]$ (contained in $B_{1}$ ) and the union of $\mathscr{T}_{c}$ with $\left[A^{\prime}, b^{\prime}\right]$ in $B_{3}$ (see Figure 6.3). Thus we study 2-shock curves based on these two curves.

For $U_{l}=\left(u_{l}, v_{l}\right)$ lying on part of $B_{1}\left(u>0, v_{l}=\rho\right)$ a segment of $\mathscr{W}_{1}\left[U_{l}\right]$ also lies on $B_{1}$ (see Figure 5.1). In this limit of $\mathscr{W}_{1}\left[U_{l}\right]$ from Figure 6.3, $U_{n}$ coincides with $D, b^{\prime}$ coincides with $K$ and the map $\mathscr{T}_{c}$ takes the segment [ $D, C]$ of $\mathscr{W}_{1}\left[U_{l}\right]$ into the segment $[C, K]$ of $B_{1}$. The latter assertion can be verified utilizing Hamiltonian systems in [23].

The proofs of the following lemmas follow from equations (2.1) and (3.1).

Lemma 1. Let $U_{m}=\left(u_{m}, \rho\right)$ be a state on $B_{1}$ with $-\sqrt{3} \rho \leq u_{m} \leq \sqrt{3} \rho$. Then the Hugoniot curve from $U_{m}$ is formed by the straight line $v=\rho$ and by the hyperbola in $(u, v)$ given by $\left(3 u-u_{m}\right)^{2}-3(v+\rho)^{2}=4 u_{m}^{2}-12 \rho^{2}$. Moreover, this hyperbola intersects $B_{1}$ at $U_{m}$ and $U_{m}^{\prime}=\left(-u_{m} / 3, \rho\right)$.

Remark 1. If $u_{m}^{2}=3 \rho^{2}$ (the vertices of the triangle), then the hyperbola mentioned in Lemma 1 degenerates into the two straight lines $\left(3 u-u_{m}\right)^{2}-3(v+\rho)^{2}=$ 0 .

Lemma 2. Let $U_{m_{1}}=(-l, \rho)$ and $U_{m_{2}}=(l, \rho)$ be two intermediate states on $B_{1}$ with $0 \leq l \leq \sqrt{3} \rho$. Then the hyperbola branches contained in the Hugoniot curves based on $U_{m_{1}}$ and on $U_{m_{2}}$ intersect each other at the point $f=\left(0,-\rho+\sqrt{4 \rho^{2}-l^{2}}\right)$, for each $l$.

Remark 2. In Lemma 2, the parameter $l$ is the distance from $U_{m_{1}}$ or $U_{m_{2}}$ to $(0, \rho)$. Also, the points $f$ lie on the $v$-axis.

Consider the Hugoniot curve based on $U_{m_{1}}$. Two sets of points $U$ on this curve represent 2-shocks $\left(U_{m_{1}}, U\right)$ : the segment $\left[U_{m_{1}}, U_{m_{1}}^{\prime}\right]$ and the hyperbola segment [ $U_{m_{1}}^{\prime}, f$ ]. Similarly, the 2-shocks in the Hugoniot curve based on $U_{m_{2}}$ are the points in the segment [ $\left.U_{m_{2}}, f\right]$.

The 2-shocks in the segments [ $U_{m_{1}}, U_{m_{1}}^{\prime}$ ] and $\left[U_{m_{2}}, f\right]$ are admissible, while those in $\left[U_{m_{1}}^{\prime}, f\right]$ are inadmissible. In fact, the following remark holds.

Remark 3. Consider the Hugoniot curve based on $U_{m_{1}}$. The states $U_{*}$ in the hyperbola between $U_{m_{1}}^{\prime}$ and $f$ represent inadmissible shocks, i.e., no orbit $\mathscr{X}_{s}$ joins $U_{m_{1}}$ to $U_{*}$. In fact, $U_{m_{1}}$ is a saddle, but its unstable manifold lies on $B_{1}$ and does not reach $U_{*}$. However, this segment is the limit when $U_{m_{1}}^{*}$ tends to $U_{m_{1}}$ of admissible 2-shocks lying on Hugoniot curves based on $U_{m_{1}}^{*}$ below $B_{1}$.

To describe the folding mechanism, we need to understand the behavior of shock curves in a space of dimension higher than 2. Setting $z=l+\sqrt{3} \rho$ $(0 \leq l \leq \sqrt{3} \rho)$ where $l$ is the variable introduced in Lemma 2 to parametrize base points of Hugoniot curves, the 2-shock curves from $U_{m_{1}}$ and those from $U_{m_{2}}$ generate a pair of surfaces in the space $(u, v, z)$. The surface $M_{1}$ associated to $U_{m_{1}}$ is formed by the union $M_{11} \cup M_{12}$ of the trapezoid $M_{11}$ : $\left\{v=\rho\right.$, with $z-2 \sqrt{3} \rho \leq u \leq \frac{2 \sqrt{3} \rho-z}{3}$ and $\left.\sqrt{3} \rho \leq z \leq 2 \sqrt{3} \rho\right\}$ and the surface $M_{12}: F_{1}(u, v, z)=(3 u-z+2 \sqrt{3} \rho)^{2}-3(v-\rho)^{2}-4(z-2 \sqrt{3} \rho)^{2}+12 \rho^{2}=$ 0 , with $\sqrt{3} u \leq v \leq \rho$ and $0 \leq u$. The surface $M_{2}$ associated to $U_{m_{2}}$ is $F_{2}(u, v, z)=(3 u+z-2 \sqrt{3} \rho)^{2}-3(v-\rho)^{2}-4(z-2 \sqrt{3} \rho)^{2}+12 \rho^{2}=0$, with $-\sqrt{3} u \leq v \leq \rho, \sqrt{3} u-2 \rho \leq v$ and $0 \leq u$. The surfaces $M_{1}$ and $M_{2}$ join 
at the line $\left(0,-\rho+\sqrt{4 \rho^{2}-(z-2 \sqrt{3} \rho)^{2}}, z\right)$. Level curves of these surfaces are 2-shock curves.

The projection of $M_{1}$ on the $(u, v)$-plane consists of the segment $[K, C]$ on $B_{1}$, the triangle $\mathbf{T}$ bounded by $B_{1}$, the $v$-axis and the branch of the Hugoniot curve based on $K$, which is a straight line parallel to $B_{2}$. The projection of $M_{2}$ on the $(u, v)$-plane is the quadrilateral bounded by $B_{1}, B_{2}$, the $v$-axis and the branch of the Hugoniot curve based on $D$ which is a line parallel to $B_{3}$. The intersection of these two projections is the triangle $T$.

As we will see, $\mathbf{T}$ is also contained in the projection of the surface $M_{3}$, which we introduce below. Thus right states $U_{r}$ in $\mathbf{T}$ may be reached from $U_{l}$ by their different Riemann solution constructions.

We will now construct the surface $M_{3}$, generated by 2-shock curves based on $U_{m_{3}}$ lying on a segment $K Q$ of $B_{3}$. Here $Q$ is the point where $B_{3}$ and the boundary of the elliptic region are tangent. The position of $U_{m_{3}}$ is parametrized in a similar way as we parametrized the position of $U_{m_{2}}$; we parametrize $U_{m_{3}}$ by its distance from $Q$. Therefore

$$
U_{m_{3}}=\left(-\frac{z+\sqrt{3} \rho}{2}, \frac{\sqrt{3} z-\rho}{2}\right)
$$

where $0 \leq z \leq \sqrt{3} \rho$.

As for $U_{m_{2}}$, the 2-shock curves from $U_{m_{3}}$ also define a surface $M_{3}$ in the $(u, v, z)$ space, given by $F_{3}(u, v, z)=0$, where

$$
F_{3}=\left(\frac{3}{2} u-\frac{3 \sqrt{3}}{2} v+z\right)^{2}-3\left(\frac{\sqrt{3}}{2} u+\frac{1}{2} v-\rho\right)^{2}+12 \rho^{2}-4 z^{2}
$$

with $-\sqrt{3} u-2 \rho \leq v \leq \rho, u \leq \sqrt{3} v$ and $\sqrt{3} u \leq v$. The $(u, v)$-projection of $M_{3}$ is the quadrilateral bounded by the loci $B_{1}, B_{3}$, the segment [ $\left.O, Q\right]$ and the branch of the Hugoniot curve from $K$ which is parallel to $B_{2}$. Again, the triangle $\mathbf{T}$ is contained in this region.

It is easy to see that $M_{1}$ joins $M_{3}$ at $z=\sqrt{3} \rho$. This junction is the union of the segments $(u, \rho, \sqrt{3} \rho),-\sqrt{3} \rho \leq u \leq \frac{\rho}{\sqrt{3}}$, and $(u, \sqrt{3} u, \sqrt{3} \rho), 0 \leq u \leq$ $\frac{\rho}{\sqrt{3}}$.

So, for $z$ varying from 0 to $2 \sqrt{3} \rho$, the 2-shock curves form three smooth surfaces in $(u, v, z)$ space, which join continuously at certain curves. The composite surface has non-smooth folds at these curves. The triangle $\mathbf{T}$ is the intersection of the $(u, v)$-projections of these surfaces.

The composite surface constructed in this section is a limit of the surface in Figure 6.6 constructed in the previous section, when $U_{l}$ tends to $B_{2}$. The points $U$ are similar limits of points $U_{+}$. The triangle $\mathbf{T}$ is the limit of region $\mathscr{A}$. The curve $\mathscr{N}_{c}$ tends to the edge $O C$ of $\mathbf{T}$, while the envelope tends to the other two edges of $\mathbf{T}$.

\section{ACKNOWLEDGMENTS}

We are grateful to Professors J. Glimm, E. Isaacson, C. F. Palmeira, B. Plohr and J. Sotomayor for helpful discussions. A. Azevedo thanks the Instituto de Matemática Pura e Aplicada (IMPA) and the Pontifícia Universidade Católica do Rio de Janeiro (PUC-Rio) for hospitality. Finally we thank the Conselho 
Nacional de Desenvolvimento Científico e Tecnológico (CNPq) and the Financiadora de Estudos e Projetos (FINEP) for partial support.

\section{REFERENCES}

1. A. A. Andronov, A. A. Vitt, and S. E. Khaikin, Theory of oscillations, Addison-Wesley, Reading, MA, 1966.

2. A. V. Azevedo and D. Marchesin, Multiple viscous profile Riemann solutions in mixed elliptic-hyperbolic models for flow in porous media, IMA Vol. Math. Appl., vol 27, SpringerVerlag, New York and Berlin, 1990, pp. 1-17.

3. A. V. Azevedo, Multiple fundamental solutions in elliptic-hyperbolic systems of conservation laws, Ph. D. Thesis (in Portuguese), Pontifícia Universidade Católica do Rio de Janeiro, Rio de Janeiro, Brazil, 1991.

4. J. Bell, J. Trangenstein, and G. Shubin, Conservation laws of mixed type describing threephase flow in porous media, SIAM J. Appl. Math. 46 (1986), 1000-1017.

5. S. Canic and B. Plohr, Shock wave admissibility for quadratic conservation laws, Proc. XVII Colóquio Brasileiro de Matemática, IMPA, 1991, pp. 199-216.

6. C. Chicone, Quadratic gradients on the plane are generically Morse-Smale, J. Differential Equations 33 (1979), 159-166.

7. J. Glimm and D. Sharp, An S-matrix theory for classical nonlinear physics, Found. Phys. 16 (1986), 125-141.

8. C. C. Conley and J. A. Smoller, Viscosity matrices for two-dimensional nonlinear hyperbolic systems, Comm. Pure Appl. Mat. 22 (1970), 867-884.

9. R. Courant and K. O. Friedrichs, Supersonic flow and shock waves, Interscience, New York, 1948.

10. F. J. Fayers and J. D. Matthews, Evaluation of normalized Stone's methods for estimating three-phase relative permeabilities, Soc. Petrol. Engin. J. 24 (1984), 225-232.

11. I. M. Gel'fand, Some problems in theory of quasilinear equations, Uspekhi Mat. Nauk 14 (1959), 87-158.

12. __ Some problems in theory of quasilinear equations, Amer. Math. Soc. Transl. Ser. 2, vol. 29, Amer. Math. Soc., Providence, RI, 1963, pp. 295-381.

13. H. Holden, On the Riemann problem for a prototype of a mixed type conservation law, Comm. Pure Appl. Math. 40 (1987), 229-264.

14. E. Isaacson, D. Marchesin, and B. Plohr, Transitional waves for conservation laws, SIAM J. Math. Anal. 21 (1990), 837-866.

15. E. Isaacson, D. Marchesin, B. Plohr, and B. Temple, The classification of solutions of quadratic Riemann problems I, SIAM J. Appl. Math. 48 (1988), 1009-1032.

16. E. Isaacson and J. B. Temple, The structure of asymptotic states in a singular system of conservation laws, Adv. Appl. Math. 11 (1990), 205-219.

17. P. Lax, Hyperbolic systems of conservation laws II, Comm. Pure Appl. Math. 19 (1957), 537-566.

18. D. Marchesin and P. J. Paes-Leme, A Riemann problem with bifurcation in gas dynamics, J. Comput. Math. Appl. 12 A (1986), 433-455.

19. C. F. Palmeira, Line fields defined by eigenspaces of derivatives of maps from the plane to itself, Proc. VI International Conference of Differential Geometry, Univ. Santiago de Compostela, Spain, 1988, pp. 177-205.

20. O. A. Oleinik, On the uniqueness of generalized solution of Cauchy problem for non linear system of equations occurring in mechanics, Uspekhi Mat. Nauk (Russian Math. Surveys) 12 (1957), 169-176.

21. D. Shaeffer, M. Shearer, D. Marchesin, and P. Paes-Leme, Solution of Riemann problem for a prototype $2 \times 2$ system of non-strictly hyperholic conservation laws, Arch. Rational Mech. Anal. 97 (1987), 299-320. 
22. H. Whitney, On singularities of mappings Euclidean spaces: I-mapping from the plane to the plane, Ann. of Math. (2) 62 (1955), 347-410.

23. Ye Yan-Qian et al., Theory of limit cycles, Transl. Math. Monographs, Amer. Math. Soc., Providence, RI, 1984.

24. K. Zumbrum, B. Plohr, and D. Marchesin, Scattering behavior of transitional shock waves, Mat. Contemp. 3 (1992), 191-209.

Departamento de Matemática, Universidade de Brasília, 70.910-900, Brasilia - DF, BRAZIL

E-mail address: arthur@mat .unb.br

Instituto de Matemática Pura e Aplicada, Est. D. Castorina 110, 22460-320, Rio de JANEIRO - RJ, BRAZIL

E-mail address: marchesiQimpa.br 